\title{
Aspectos dietarios de Alsodes coppingeri Günther, 1881 (Anura: Alsodidae) en Chile
}

\section{Dietary aspects of Alsodes coppingeri Günther, 1881 (Anura: Alsodidae) in Chile}

\author{
Nicza Alveal ${ }^{1,2, *}$, Helen Díaz-Páez ${ }^{1}$, Ana Henríquez ${ }^{1}$ \& Olivia Vergara ${ }^{3}$ \\ 1'Departamento de Ciencias Básicas, Campus Los Ángeles, Universidad de Concepción. Casilla 341, Los Ángeles, Chile. \\ ${ }^{2}$ Departamento de Zoología, Campus Concepción, Universidad de Concepción. Casilla Casilla 160-C, Concepción, Chile. \\ ${ }^{3}$ School of Biological Sciences, Victoria University of Wellington, PO Box 600, New Zealand. \\ *E-mail: niczalveal@udec.cl
}

\begin{abstract}
RESUMEN
Los estudios relacionados con la composición dietaria de los anuros presentes en Chile son un campo escasamente explorado y que permiten aportar con conocimiento sobre la biología de las especies. Alsodes coppingeri habita los arroyos de montaña de los bosques templados de Nothofagus de la Patagonia chilena. Estos anuros han sido encontrados bajo troncos en los márgenes de arroyos. Para este estudio se analizaron 44 especímenes de $A$. coppingeri provenientes de Laguna Caiquenes, Caleta Tortel y Villa O’Higgins (Región de Aysén, Chile). Los ítems presa fueron clasificados a nivel ordinal y se estimó la frecuencia de ocurrencia, de abundancia e índice de importancia relativa (IRI). Se determinó un $70,5 \%$ de frecuencia de ocurrencia, donde la dieta de A. coppingeri estuvo compuesta principalmente de artrópodos. Las presas más frecuentes correspondieron a coleópteros, colémbolos, dípteros, arañas e isópodos, con un notorio predominio de estados larvales de coleópteros, dípteros y lepidópteros.
\end{abstract}

Palabras claves: Rana de pecho espinoso austral, anfibios, dieta, artrópodos.

\begin{abstract}
The studies related to dietary composition of anurans present in Chile are a field sparsely explored and allow to contribute with knowledge about the biology of the species. Alsodes coppingeri inhabits mountain streams in Nothofagus temperate forests, of Chilean Patagonia. These frogs have been found under logs in stream margins. In this study, 44 specimens of $A$. coppingeri from Laguna Caiquenes, Tortel and Villa O'Higgins (Aysen Region, Chile) were analyzed. The prey items were ranked at the ordinal level. The frequency of occurrence percentage and abundance of prey items and relative importance index (IRI) were estimated. The frequency of occurrence percentage was $70.5 \%$, where the diet of $A$. coppingeri was mainly composed of arthropods. The most important preys were beetles, springtails, flies, spiders and isopods, with an obvious predominance of larval stages of beetles, flies and moths.
\end{abstract}

KEYWORDS: Southern spiny frog chest, amphibians, diet, arthropods.

\section{INTRODUCCIÓN}

Los anfibios son un grupo diverso de vertebrados, para los cuales se ha determinado una dieta de tipo oportunista y se ha descrito que tanto sapos como ranas solo atrapan presas en movimiento (Browne 2009). La conducta alimentaria de los anfibios ha sido foco de numerosos estudios y ha definido su rol en los ecosistemas terrestres y acuáticos (Cobas \& Arbib 1992, Lajmanovich 1996, Caldwell 1996, Muñoz-Guerrero et al. 2007, Arroyo et al. 2008), donde actúan como depredadores capaces de controlar muchas poblaciones de organismos, especialmente de invertebrados
(Wells 2007, Toledo et al. 2007). Junto a esto, se ha detectado como principales ítems alimentarios a moluscos, anélidos, ciempiés, milpiés, arácnidos, crustáceos y principalmente insectos (Duellman \& Trueb 1994, Santos et al. 2004).

El estudio de la ecología trófica ha provisto información fundamental para comprender el rol de estos organismos en el ambiente, proporcionando datos acerca de la historia de vida y patrones conductuales (Wells 1978, Murphy 1992, Stebbins \& Cohen 1995). Es así, como recientes estudios han puesto en duda el carácter generalista de la conducta trófica de los anfibios, donde los análisis dietarios realizados 
en dendrobátidos y bufónidos han determinado una dieta especializada en ciertas categorías de presas, entre las que destacan las hormigas (Hymenoptera) y termitas (Isoptera) (Donnelly 1991, Caldwell 1996, Méndez-Narváez et al. 2014).

La dieta se ve influenciada por múltiples factores, siendo los más importantes los cambios ambientales (Solé et al. 2009), el tamaño corporal de los individuos (Lima 1998, Batista et al. 2011, Sugai et al. 2012), la estacionalidad (Maragno \& Souza 2011) y estrategias de caza (Maneyero et al. 2004). Por lo tanto, la dieta se transforma en un importante componente de la historia natural de las especies y sugiere consecuencias ecológicas (Anderson \& Mathis 1999), que han llevado a plantear una relación directa entre las condiciones ambientales, las alteraciones de los hábitats y la distribución de las presas (Parker \& Goldstein 2004). Basados en este vínculo ambiental, Batista et al. (2011) reconocen que la información acerca de la dieta de las especies puede ayudar a establecer estrategias de conservación.

En Chile, la información acerca de los hábitos alimentarios de anfibios es escasa e incompleta (Vidal \& Labra 2008), con tan solo unos pocos registros que hacen referencia a consumos puntuales (Cei 1962, 1980) o incorporando análisis tróficos, por ejemplo, para las especies de Pleurodema thaul, Pleurodema bufoninum, Rhinella spinulosa y Batrachyla taeniata (Valencia et al. 1982, Núñez et al. 1982, PincheiraDonoso 2002a, 2002b, Díaz-Páez \& Ortiz 2003a, Gutiérrez et al. 2008).

Alsodes coppingeri es una especie típica de la Patagonia chilena que habita en arroyos de montaña, bajo rocas y troncos de árboles muertos de los bosques templados, entre las regiones de Aysen y Punta Arenas (Formas et al. 2008, Cisternas et al. 2013).

El objetivo del presente trabajo fue describir la composición dietaria de A. coppingeri identificando las presas y estableciendo la abundancia $(\mathrm{N})$, frecuencia de ocurrencia (F) e índice de importancia relativa (IRI) en la dieta de los adultos de $A$. coppingeri provenientes de la Región de Aysén, Chile.

\section{MATERIALES Y MÉTODOS}

Se colectaron 44 ejemplares de A. coppingeri (permiso del Servicio Agrícola Ganadero (Resolución 6494/2006), los que fueron encontrados bajo troncos y piedras en los bosques, a lo largo del camino y en la ribera de arroyos montañosos (Formas et al. 2008). Las colectas se realizaron en forma manual durante los meses de primavera-verano de los años 2006-2007 en los sectores de Laguna Caiquenes

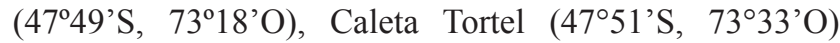
y Villa O'Higgins $\left(48^{\circ} 22^{\prime} \mathrm{S}, 7^{\circ} 29^{\prime} \mathrm{O}\right)$ (Fig. 1). Estas localidades se caracterizan por poseer una vegetación de matorral arborescente caducifolio templado de Nothofagus antarctica y Berberis microphylla, junto a zonas de bosque siempreverde templado interior compuesto de Nothofagus betuloides, Desfontainia spinosa y Drimys winteri (Luebert \& Pliscoff 2006).

Los especímenes fueron eutanasiados con benzocaina al $1 \%$ y fijados en formalina al $10 \%$ para su posterior preservación en etanol $70 \%$. Todos los ejemplares fueron depositados en el Museo de Zoología de la Universidad de Concepción (MZUC 34440-34442, 34444, 34449-34452, 34455, $34457,34473,34475,34517-34518,34521-34523,36555$, 36557-36561, 36583; 41772-41791). Para la obtención de los contenidos estomacales se realizó la disección de los individuos extrayendo los estómagos desde el cardias al píloro, los que fueron conservados en etanol $70^{\circ}$. Las muestras fueron analizadas en laboratorio bajo una lupa estereoscópica Olympus SZ51.

Los ítems de invertebrados fueron determinados hasta la menor categoría taxonómica posible utilizando las descripciones de Peña (1988) y Artigas (1994a, 1994b), mientras que los vegetales sólo fueron considerados como restos sin determinar. Para cada ítem alimenticio se registró el número total y se procedió a determinar la valoración volumétrica $(\mathrm{W})$ mediante el cálculo del volumen de líquido desplazado en una probeta de $0,01 \mathrm{~mL}$ de precisión. Con estos datos se calculó el porcentaje agregado de volúmenes y el número de ítems de consumo para cada especie (Litvaitis et al. 1994). Posteriormente, se determinó un índice de importancia relativa (IRI) (Powell et al. 1990) para cada taxón de invertebrados, para lo cual se utilizó la siguiente fórmula:

$$
\text { IRI }=\% \mathbf{N}+\% \mathbf{F}+\mathrm{W} / \mathbf{3}
$$

Donde $\% \mathrm{~N}$ corresponde al porcentaje numérico agregado de los ítems alimenticios, \%F es la frecuencia de ocurrencia porcentual y W corresponde al volumen agregado para cada ítem.

Con los valores de IRI se establecieron tres categorías de importancia alimentaria: primaria, secundaria y terciaria, donde los valores más altos del índice indican la mayor importancia del ítem alimentario. Dado que no se trata de variables continuas, para fijar los límites de cada categoría, se priorizó el valor de la mediana $(2,56)$ sobre el valor de la media $(3,62 \pm 3.19)$ sumada a la desviación estándar (DíazPáez \& Ortiz 2003b). Adicionalmente, se realizó el test de Kruskal-Wallis para determinar diferencias en los ítems de consumo, lo que indicaría una preferencia de unos por sobre los otros (Siegel \& Castellan 1988). 


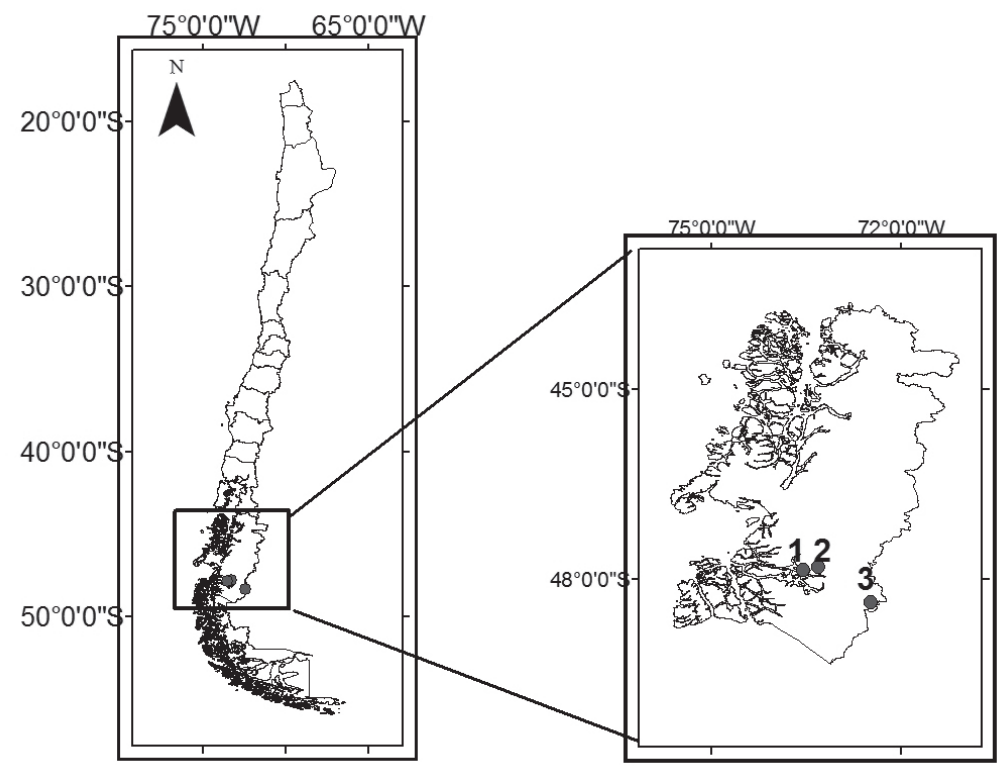

Figura 1. Ubicación geográfica de la zona de estudio. 1. Caleta Tortel, 2. Laguna Caiquenes y 3. Villa O’Higgins, Región de Aysén, Chile.

Figure 1. Geographic location of the survey area. 1. Caleta Tortel, 2. Laguna Caiquenes and 3. Villa O'Higgins, Aysén Region, Chile.

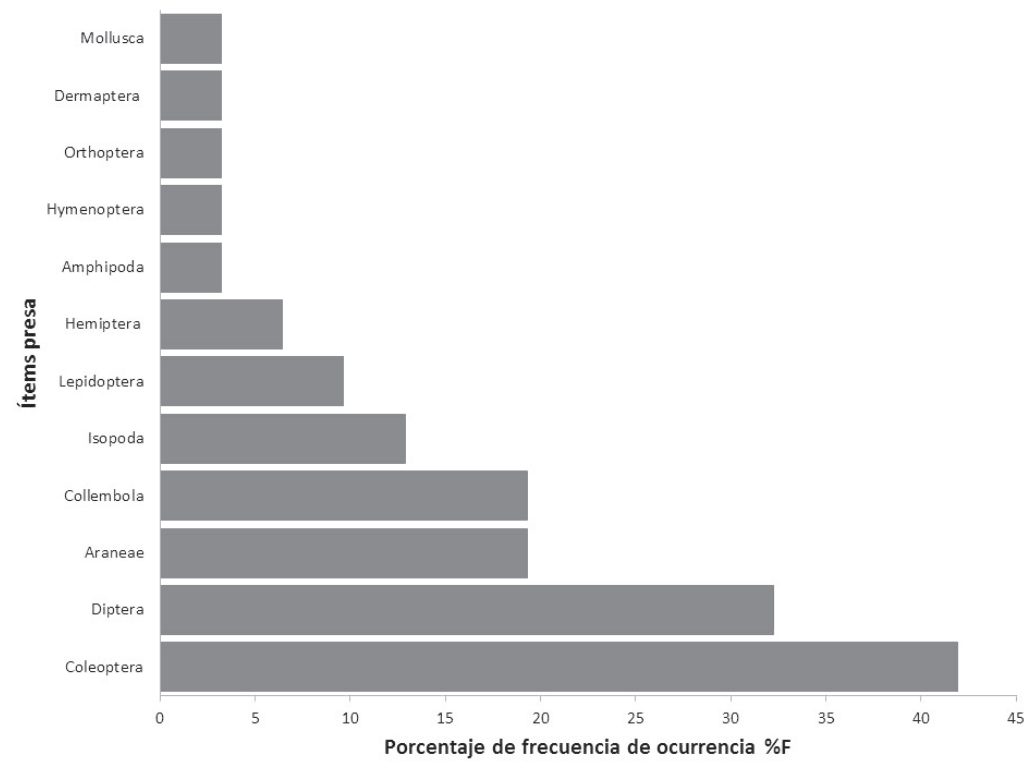

FIgURA 2: Porcentaje de frecuencia de ocurrencia de los OTUs (Unidad Taxonómica Operacional).

FIGURE 2: Frequency of occurrence percentage of OTUs (Operational Taxonomic Units).

\section{RESULTADOS}

De los 44 ejemplares de $A$. coppingeri analizados, 31 presentaron contenido estomacal constituyendo una alta frecuencia de ocurrencia, la que alcanza un 70,5\%. Se determinó un total de 66 presas, contenidas en 22 categorías taxonómicas todas correspondientes a la fracción animal (Tabla 1). Con la agrupación de éstas siguiendo el criterio de "Unidad Taxonómica Operacional" (OTU) se consideraron 12 ítems presa: Coleoptera, Collembola,
Diptera, Dermaptera, Hemiptera, Hymenoptera, Isopoda, Lepidoptera, Orthoptera, Amphipoda, Aranea y Mollusca (Sanabria et al. 2007) (Tabla 1). En el análisis estomacal se determinó la presencia de un bajo consumo de detritos y restos vegetales, los cuales no fueron identificados. Los detritos consistieron en pequeños gránulos de minerales y los vegetales incluyeron trozos de tallos, hojas y semillas indeterminadas. Así, nuestros resultados muestran que A. coppingeri posee un consumo mayoritario de ítems animales, entre los que resultan más abundantes los OTUs 
Coleoptera, Diptera, Collembola y Araneae (Fig. 2) que en conjunto constituyen un IRI de un $70 \%$ de la dieta total de esta especie. Para Coleoptera destaca el consumo de especímenes de las familias Scarabaeidae, Curculionidae, Elateridae y Carabidae, mientras que para Diptera las familias con mayor abundancia corresponden a Tipulidae y Piophilidae (Tabla 1).

Adicionalmente, se observa un consumo de adultos y estadios larvales de los OTUs Lepidoptera e Hymenoptera, predominando el consumo de tipúlidos en ambos estadios (12,9\% adultos y $6,25 \%$ larvas).
Los ítems presa considerados como primarios en la dieta de $A$. coppingeri corresponden a las familias Sminthuridae $(13,95)$, Oniscidae $(9,18)$ y presas del orden Araneae $(9,86)$ La dominancia de los Sminthuridae está dada por el número de individuos encontrados en los estómagos, sin embargo, en los otros ítems considerados primarios la dominancia está dada principalmente por el volumen de las presas (Tabla 1). El test de Kruskal-Wallis $(\mathrm{H}=10,23$ y $\mathrm{P}=0,44)$ no revela diferencias significativas en el consumo de los ítems alimenticios, por lo que no se puede evidenciar que $A$. coppingeri discrimine frente al consumo de algunos OTUs por sobre los otros.

TABLA 1: Abundancia y frecuencia de los ítems presa presentes en el contenido estomacal de $A$. coppingeri. \%N: porcentaje numérico agregado. \%F: frecuencia de Ocurrencia porcentual, W: Volumen e IRI: Índice de Importancia Relativa.

TABLE 1: Abundance and frequency of prey items found in the stomach contents of A. coppingeri. \% N: Numerical percentage. \% F: Frequency of occurrence percentage, W: Volumetric percentage and IRI: Index of Relative Importance.

\begin{tabular}{|c|c|c|c|c|c|}
\hline CATEgoría OTUS & $\% \mathbf{N}$ & $\% \mathrm{~F}$ & $\mathbf{W}$ & IRI & Clasificación \\
\hline \multicolumn{6}{|l|}{ Coleoptera } \\
\hline Curculionidae & 3,03 & 6,45 & 0,08 & 3,24 & \multirow{4}{*}{ Terciario } \\
\hline Elateridae & 3,03 & 6,45 & 0,04 & 3,22 & \\
\hline Elateridae(larva) & 1,51 & 3,23 & 0,04 & 1,62 & \\
\hline Carabidae & 3,03 & 6,45 & 0,06 & 3,23 & \\
\hline Scarabaeidae & 4,54 & 9,68 & 0,50 & 4,98 & Secundario \\
\hline Pselaphidae & 1,51 & 3,23 & 0,001 & 1,60 & \multirow{3}{*}{ Terciario } \\
\hline Indeterminado 1 & 3,03 & 3,23 & 2,04 & 2,81 & \\
\hline Indeterminado 2 & 3,03 & 3,23 & 0,04 & 1,62 & \\
\hline \multicolumn{6}{|l|}{ Diptera } \\
\hline Piophilidae & 3,03 & 6,45 & 0,04 & 3,22 & \multirow{5}{*}{$\begin{array}{c}\text { Terciario } \\
\text { Secundario } \\
\text { Terciario }\end{array}$} \\
\hline Tipulidae & 6,06 & 12,90 & 0,04 & 6,43 & \\
\hline Tipulidae (larva) & 4,54 & 6,45 & 0,27 & 1,69 & \\
\hline Indeterminado 1 & 3,03 & 3,23 & 0,50 & 2,30 & \\
\hline Indeterminado 2 & 1,51 & 3,23 & 0,02 & 1,61 & \\
\hline \multicolumn{6}{|l|}{ Collembola } \\
\hline Sminthuridae & 21,21 & 19,35 & 0,28 & 13,95 & Primario \\
\hline \multicolumn{6}{|l|}{ Dermaptera } \\
\hline Forficulidae & 1,51 & 3,23 & 0,07 & 1,63 & \multirow{5}{*}{ Terciario } \\
\hline Hemiptera & 3,03 & 6,45 & 0,06 & 3,23 & \\
\hline Lepidoptera & & & & & \\
\hline Geometridae(larva) & 3,03 & 6,45 & 0,34 & 3,32 & \\
\hline Indeterminado 3 (larva) & 1,51 & 3,23 & 0,04 & 1,62 & \\
\hline Hymenoptera (larva) & 1,51 & 3,23 & 0,001 & 1,60 & \\
\hline Orthoptera & 1,51 & 3,23 & 0,03 & 1,61 & \\
\hline \multicolumn{6}{|l|}{ Isopoda } \\
\hline Oniscidae & 13,64 & 12,90 & 0,36 & 9,18 & Primario \\
\hline Amphipoda & 1,51 & 3,23 & 0,03 & 1,61 & Terciario \\
\hline Araneae & 9,09 & 19,35 & 0,69 & 9,86 & Primario \\
\hline Mollusca & 1,51 & 3,23 & 0,05 & 1,62 & Terciario \\
\hline
\end{tabular}




\section{DISCUSIÓN}

El análisis trófico en $A$. coppingeri muestra una tendencia al consumo de taxa, tanto de organismos terrestres como acuáticos (coleópteros, dípteros, colémbolos, arácnidos, isópodos, etc.). La dominancia numérica de los Sminthuridae en la dieta podría deberse a que este taxón constituye un componente fundamental de la fauna de suelo de los bosques australes, sin embargo sin un análisis de oferta ambiental no es posible aseverar a cabalidad este aspecto.

El análisis de los restos ha mostrado que al igual que en otros anuros (Pincheira-Donoso 2002a, 2002b, Díaz-Páez \& Ortiz 2003a, Gutiérrez et al. 2008) existe mayor variedad en los ítems presa para coleópteros y dípteros, lo que se traduce en una mayor diversidad de familias incorporadas en la dieta, destacando la presencia de tipúlidos en estado adulto y larvario. Este consumo mayoritario de tipúlidos se debe particularmente a que son presas grandes y nocturnas, lo cual coincide con los períodos máximos diarios de forrajeo y actividad reproductiva de estos anuros (Díaz-Páez \& Ortiz 2003a).

Así mismo, la variedad de ítems presa sugiere que se trata de un anfibio que obtiene su alimento en distintos hábitats, lo que es sustentado por las descripciones ecológicas de la especie (Cisternas et al. 2013). Los resultados indican que este anfibio no discrimina entre los diferentes ítems alimenticios, lo que lleva a que $A$. coppingeri se alimente principalmente de organismos invertebrados de diversos taxa.

Se ha detectado que tanto los atributos de tamaño, movimiento, palatabilidad y valor nutritivo pueden afectar la selección que hacen los anuros del alimento (Anderson \& Mathis 1999, Anderson et al. 1999). Sin embargo, sin datos disponibles acerca de la oferta ambiental, no es posible referirse a una selección de los ítems alimentarios determinados con mayor valor de importancia (Whittaker et al. 1977, Anderson et al. 1999).

Por último, la ingestión de restos vegetales es común en anuros (Camera et al. 2014). Las hipótesis que explicarían esta conducta son: 1) la ingesta posee una función de limpieza intestinal al ayudar en la eliminación de parásitos intestinales o los exoesqueletos de los artrópodos, 2) al aporte adicional en nutrientes y agua que proveerían estos recursos (Anderson et al. 1999) y 3) por captura incidental durante la ingesta del alimento (Whittaker et al. 1977). En nuestro estudio, debido al bajo consumo de vegetales por parte de $A$. coppingeri no es posible confirmar ninguna de estas hipótesis por lo que será necesario evaluar a futuro, la probable función que cumple este ítem alimentario para esta especie.

\section{AGRADECIMIENTOS}

Los autores agradecen al Servicio Agrícola y Ganadero (SAG, permiso de colecta $\mathrm{N}^{0}$ 6494, Diciembre 2006). Está investigación fue patrocinada por el Proyecto DIUC 210.412.045-1sp de la Universidad de Concepción.

\section{BIBLIOGRAFÍA}

Anderson, A., Haukos, D. \& Anderson, J. 1999. Diet composition of three anurans from the Playa wetlands of northwest Texas. Copeia 1999: 515-520.

Anderson, M. \& Mathis, A. 1999. Diets of two sympatric Neotropical Salamanders, Bolitoglossa mexicana and $B$. rufescens, with notes on reproduction for $B$. rufescens. Journal of Herpetology 33: 601-607.

Arroyo, S., Serrano-Cardozo, V. \& Ramírez, M. 2008. Diet, microhabitat and time of activity in a Pristimantis (Anura: Strabomantidae) assemblage. Phyllomedusa 7: 109-119.

Artigas, J.N. 1994a. Entomología Económica. Insecta de Interés Agrícola, Forestal, Médico y Veterinario (Nativos, Introducidos y Susceptibles de ser Introducidos). Vol. 1. Ediciones Universidad de Concepción, Concepción. $1126 \mathrm{pp}$.

Artigas, J.N. 1994b. Entomología Económica. Insecta de Interés Agrícola, Forestal, Médico y Veterinario (Nativos, Introducidos y Susceptibles de ser Introducidos). Vol. 2. Ediciones Universidad de Concepción, Concepción. 943pp.

Batista, R.C., De-Carvalho, C.B., Freitas, E.B., Franco, S.C., Batista, C.C., Coelho, W.A. \& Faria, R.G. 2011. Diet of Rhinella schneideri (Werner, 1894) (Anura: Bufonidae) in the Cerrado, Central Brazil. Herpetology Notes 4: 17-21.

Browne, R.K. 2009. Amphibian diet and nutrition. AArk Science and Research. <http://www.amphibianark.org/pdf/ Husbandry/> Amphibian\%20diet\%20nutrition.pdf

Caldwell, J. 1996. The evolution of Myrmecophagy and its correlation in poison frogs (Family Dendrobatidae). Journal of Zoology (London) 240: 75-101.

CAmera, B., Krinski, D. \& CAlvo, I. 2014. Diet of the Neotropical frog Leptodactylus mystaceus (Anura: Leptodactylidae). Herpetology Notes 7: 31-36.

Cei, J. 1962. Batracios de Chile. Ediciones Universidad de Chile, Santiago. 128pp.

CeI, J.1980. Amphibians of Argentina. Monitore Zoologico Italiano (N.S.) Monographies 2:1-609.

Cisternas, J., Correa, C., Velásquez, N. \& Penna, M. 2013. Reproductive features of Chaltenobatrachus grandisonae (Anura: Batrachylidae) within a protected area in Patagonia, Chile. Revista Chilena de Historia Natural 86: 365-368.

Cobas, A. \& ArbiB, M. 1992. Prey-catching and predatoravoidance in frog and toad: Defining the Schemas. Journal of Theoretical Biology 157: 271-304.

Díaz-PÁez, H. \& Ortiz, J. C. 2003a. Hábitos alimentarios de Pleurodema thaul (Anura: Leptodactylidae), en Concepción, Chile. Gayana 67: 25-32.

Díaz-PÁez, H. \& Ortiz, J.C. 2003b. Evaluación del estado de conservación de los anfibios en Chile. Revista Chilena de 
Historia Natural 76: 509-525.

Donnelly, M.A. 1991. Feeding patterns of the strawberry poison frog, Dendrobates pumilio (Anura: Dendrobatidae). Copeia 1991: 723-730.

Duellman, W.E. \& Trueb, L. 1994. Biology of amphibians. Baltimore and London, John Hopkins University Press. $613 p p$.

Formas, J.R., Nuñez, J. \&. Cuevas, C. 2008. Identidad de la rana austral chilena Eupsophus coppingeri (Amphibia, Anura, Neobatrachia): Evidencias morfológicas, cromosómicas y moleculares. Revista Chilena de Historia Natural (Chile) 81: 3-20.

Gutiérrez, N., Méndez, M.A., \& Sallaberry, M. 2008. Hábitos alimentarios de Bufo spinulosus Wiegmann, 1835 (Anura: Bufonidae) en la localidad de Farellones (Región Metropolitana). Boletín del Museo Nacional de Historia Natural (Chile) 57: 141-147.

Lajmanovich, R.C. 1996. Dinámica trófica de juveniles de Leptodactylus ocellatus (Anura: Leptodactylidae), en una isla del Paraná, Santa Fe, Argentina. Cuadernos de Herpetología 10(1-2): 11-23.

LimA, A.P. 1998. The effects of size on the diets of six sympatric species of postmetamorphic litter anurans in Central Amazonia. Journal of Herpetology 32(3): 392-399. http:// dx.doi.org/10.2307/1565453

Litvaitis, J.A., Titus, K \& Anderson, E.M. 1994. Measuring vertebrates: use of terrestrial habitats and foods. En: Research and management techniques for wildlife and habitats. (Ed. Bookhout, T.A.), pp. 254-274. 5th ed. Wildlife Society, Bethesda, MD.

Luebert, F. \& Pliscoff, P. 2006. Sinopsis bioclimática y vegetacional de Chile. Santiago de Chile: Editorial Universitaria. 316pp.

Maneyro, R., Naya, D.E., da Rosa, I., Canavero, A. \& Camargo, A. 2004. Diet of the South American frog Leptodactylus ocellatus (Anura, Leptodactylidae) in Uruguay. Iheringia, Série Zoologia 94: 57-61.

Maragno, F.P. \& SouZA, F.L. 2011. Diet of Rhinella scitula (Anura, Bufonidae) in the Cerrado, Brazil: the importance of seasons and body size. Revista Mexicana de Biodiversidad 82: 879-886.

Méndez-Narváez, J. Ospina-Sarria, J. \& Bolívar, W. 2014. Diet and trophic ecology of Leptodactylus fragilis (Leptodactylidae) and Dendropsophus columbianus (Anura: Hylidae) in a disturbed area in southwestern Colombia. Herpetology Notes 7: 299-305.

Muñoz-Guerrero J., Serrano, V. \& Ramírez-Pinilla, M.P. 2007. Microhabitat use, diet and time of activity of four sympatric Neotropical hylid frogs (Anura: Hylidae). Caldasia 29(2): 413-425.

Murphy, C. 1992. The mating of the barking treefrog (Hyla gratiosa). Ph D. Dissertation, Cornell University.

NúÑEz, H., LABRA, M.A. \& NúÑEZ, J. 1982. Hábitos alimentarios de dos poblaciones andinas de Bufo spinulosus Wiegmann, 1985 (Anura: Bufonidae). Boletín Museo Nacional de Historia Natural. Santiago 39: 81-91.

Parker, M.L. \& Goldstein, M.I. 2004. Diet of Rio Grande leopard frog (Rana berlandieri) in Texas. Journal of Herpetology
38: $127-130$.

PeÑa, L. 1988. Introducción a los insectos de Chile. Editorial Universitaria, Chile. 254pp.

Pincheira-Donoso, D. 2002a. Nota sobre la alimentación de Pleurodema bufonina Bell 1843 (Anura-Leptodactylidae). Gayana 66: 77-80.

Pincheira-Donoso, D. 2002b. Dieta de Batrachyla taeniata (Girard, 1854) en poblaciones de Concepción, Chile (Anura: Leptodactylidae). Noticiario Mensual, Museo Nacional de Historia Natural 348: 3-7.

Powell R., Parmelee, J.S, Rice, M.A.,Smith, D.D. 1990. Ecological observations of Hemidactylus brooki haitianus Meerwath (Sauria: Gekkonidae) from Hispanoila. Caribbean Journal of Science 26: 67-70.

Sanabria E., Quiroga, L., \& Acosta, J.C. 2007. Hábitos alimentarios de infantiles de Pleurodema nebulosum (Anura: Leptodactylidae), en Matagusanos, San Juan, Argentina. Revista Peruana de Biología 14(2): 295-296.

Santos, E., Almeida, A. \& Vasconcelos, S. 2004. Feeding habits of six anuran (Amphibia: Anura) species in a rainforest fragment in Northeastern Brazil. Iheringia, Série Zoologia. 94(4): 433-438.

Siegel, S. \& Castellan, N.J. 1988. Nonparametrics statistica for the behavioral sciences, McGraw-Hill Book Company, New York. 399 pp.

Solé, M., Dias, I.R., Rodrigues, E.A.S., Marciano-Junior, E., Branco, S.M.J., Cavalcante, K.P. \& Rödder, D. 2009. Diet of Leptodactylus ocellatus (Anura: Leptodactylidae) from a cacao plantation in southern Bahia, Brazil. Herpetology Notes 2: 9-15

Stebbins, R. \& Cohen, N. 1995. A Natural History of Amphibians. Princeton University Press, Princeton, New Jersey. 332pp.

Sugai, J.L.M.M., Terra, J.S., \& Ferreira, V.L. 2012. Diet of Leptodactylus fuscus (Amphibia: Anura: Leptodactylidae) in the Pantanal of Miranda river, Brazil. Biota Neotropica 12: 99-104.

Toledo, L.F., Ribeiro, R.S., \& Haddad, C.F.D. 2007. Anurans as prey: an exploratory analysis and the size relationships between predators and their preys. Journal of Zoology. 271: 170-177.

Valencia J., Veloso, A. \& Sallaberry, M. 1982. Nicho trófico de las especies de herpetozoos del transecto Arica-Chungará. En: El ambiente natural y las poblaciones humanas de los Andes del norte grande de Chile (Arica, Lat. 18 28'S). (Eds. Veloso, A. \& E. Bustos-Obregón), pp. 269-291. Volumen I, Proyecto MAB-6, UNEP-UNESCO 1105-7701, ROSTLAC, Montevideo.

VidAl, M. \& Labra, M.A. 2008. Dieta de anfibios y reptiles. En: Herpetología de Chile. (Eds. Vidal, M. \& M.A. Labra), pp. 453-482. Science Verlag, Santiago, Chile.

WeLLs, K.D. 1978. Territoriality in the green frog (Rana clamitans): Vocalizations and agonistic behavior. Animal Behaviour 26: 1051-1063.

WeLLs, K.D. 2007. The ecology and behavior of amphibians. University Chicago Press, Chicago. 1148pp.

Whittaker, J.R., Rubin, J.O.D \& MunseE, J.R. 1977. Observations on food habits of four species of spadefoot toads, genus Scaphiopus. Herpetologica 33: 468-475.

Recibido: 03.06.14

Aceptado: 30.03 .15 